\title{
Modelagem estatística da precipitação mensal e anual e no período seco para o estado de Minas Gerais
}

\author{
Carlos R. de Mello ${ }^{1}$ \& Antônio M. da Silva ${ }^{1}$
}

\begin{abstract}
RESUMO
Objetivou-se, com o presente estudo, ajustar modelos lineares para predição da precipitação média mensal (no período úmido) e anual e no período seco, baseados nas coordenadas geográficas (latitude e longitude) e altitude para o Estado de Minas Gerais. Aplicaram-se dados diários de precipitação, provenientes da Agência Nacional de Águas (ANA) de 209 estações meteorológicas, das quais 197 foram usadas para ajuste dos modelos e 12 para sua validação final. O coeficiente de determinação ajustado $\left(R^{2}\right)$, o erro médio absoluto $(\%)$, a tendência das estimativas $(\%)$ e significância dos parâmetros, foram considerados na avaliação dos modelos. De maneira geral, os modelos apresentaram bons parâmetros estatísticos de validação, com $R^{2}$ maior que 0,70 , erro médio menor que $10 \%$ e tendência não significativa $(<2 \%$ em relação à média). O modelo para o período seco, por sua vez, apresentou maior superestimativa que os demais, mostrando que outras variáveis associadas aos aspectos topográficos, podem ser necessárias para um modelo mais preciso. Apesar desta situação, os modelos se apresentam em condições de aplicação prática constituindo-se em uma ferramenta importante para o manejo ambiental no Estado.
\end{abstract}

Palavras-chave: modelos estatísticos, climatologia, hidrologia, precipitação pluvial

\section{Statistical modeling of monthly, annual and dry season mean precipitation for the State of Minas Gerais}

\begin{abstract}
This study aimed at adjusting statistical linear models for prediction of total mean precipitation associated to monthly (in the wet season), annual and dry season periods, based on geographical coordinates (latitude and longitude) and altitude for the State of Minas Gerais, Brazil. Daily precipitation data from the "Agência Nacional de Águas" (ANA) for 209 pluviometric stations were applied, 197 for modeling adjustment and 12 for final validation. Coefficient of determination adjusted $\left(\mathrm{R}^{2}\right)$, mean absolute error $(\%)$, prediction bias $(\%)$ and estimated parameters significance were considered for evaluation of models. The monthly and annual precipitation models presented good statistical validation coefficients, with $\mathrm{R}^{2}$ greater than 0.70 , mean error smaller than $10 \%$ and bias not significant $(<2 \%$ in relation to mean value). However, the dry season model presented an overestimation of precipitation, showing that more variables associated to topographic characteristics would be necessary to produce a more accurate model. Nevertheless, the adjusted models present good conditions for practical applications, forming an important tool for environmental management in the State of Minas Gerais.
\end{abstract}

Key words: statistical models, climatology, hydrology, pluvial precipitation 


\section{INTRODUÇÃO}

Dentro do ciclo hidrológico, a precipitação consiste de uma variável de entrada, fundamental para o entendimento da dinâmica do meio físico. Seu entendimento e comportamento são de suma importância para estudos estratégicos associados ao planejamento do meio ambiente, geração de energia e manejo da agricultura, especialmente em condições tropicais.

Diversos estudos visando ao mapeamento da precipitação têm sido desenvolvidos com aplicação de várias técnicas, uma vez que a disponibilidade de dados climáticos e hidrológicos é pequena se comparada com grandes extensões territoriais, em especial em países em desenvolvimento (Marquínez et al., 2003; Martinez-Cob, 1996; Mello et al., 2007). Sabe-se que o fenômeno da precipitação apresenta alta variabilidade espaço-temporal, razão por que é considerado probabilístico e aleatório pela hidrologia estocástica, passível de ajuste de modelos estocásticos multivariados (Wu et al., 2006; Goodale et al. 1998; Daly et al., 1994). Comparativamente, modelos matemáticos apresentam melhor aplicabilidade que o uso de mapas temáticos que, muitas vezes, regionalizam a variável a ser mapeada na forma de intervalos de classe, não permitindo precisão específica a uma localidade.

Daly et al. (1994), Matínez-Cob (1996), Weisse \& Bois (2001) e Marquínez et al. (2003) discutem, em seus trabalhos, a influência do relevo no comportamento da precipitação e que a inclusão da altitude é indispensável na modelagem e mapeamento da chuva, evitando estimativas tendenciosas e menos precisas. Além da altitude, Marquínez et al. (2003) mencionam que a continentalidade, expressa pelas coordenadas geográficas, também são variáveis explicativas de características climáticas quantitativas, como precipitação, temperatura do ar e radiação solar.

Minas Gerais possui extensas cadeias de montanhas espalhadas por seu território e a influência orográfica nas diferentes condições climáticas do Estado é uma realidade constatada estatisticamente por Mello et al. (2007) e Viola (2006). Além deste aspecto, a variabilidade climática do Estado, função de sua localização e extensão territorial, combinada com a altitude, também reflete no seu comportamento pluvial, produzindo variabilidade importante da quantidade de chuvas, sobretudo na direção latitudinal.

Com base no fato de que a altitude, a latitude e a longitude influenciam nas condições climáticas de uma região, conclui-se ser possível, aplicando-se técnicas estatísticas de modelagem, ajustar equações lineares de regressão múltipla para estimativa dos totais médios precipitados em função das coordenadas geográficas e altitude. Ressalta-se que a variável climática em questão (precipitação), mesmo em termos médios, apresenta aleatoriedade consideravelmente alta, implicando em modelos que poderão ser de qualidade inferior aos desenvolvidos por Sediyama \& Mello Jr. (1998) para temperatura média mensal e anual, uma vez que esta possui menor variabilidade espacial e maior correlação com as coordenadas geográficas. Uma das principais aplicações deste estudo está associada à geração de balanços hídricos anuais em diferentes bacias hidrográficas, visto que existem mode- los semelhantes para estimativa da temperatura média mensal e anual do ar para o Estado de Minas Gerais.

Neste contexto se propôs, com este estudo, ajustar modelos estatísticos para estimativa dos totais precipitados médios mensais no período chuvoso (setembro - abril), do total precipitado médio no período mais seco do ano (de maio a agosto) e total médio anual, para aplicação no Estado de Minas Gerais.

\section{MATERIAL E MÉTODOS}

A base de dados aplicada a este estudo foi obtida junto à Agência Nacional de Águas - ANA (Hidroweb/ANA), consistindo de dados diários de precipitação de 209 estações pluviométricas. Na Figura 1 é possível verificar as estações aplicadas à modelagem e aquelas utilizadas estritamente para validação, distribuídas no estado de Minas Gerais e em seu entorno. Os dados foram totalizados em períodos mensais, anual e no período seco, o qual se estende de forma mais acentuada entre maio e agosto; assim, foram geradas séries históricas correspondentes aos meses de janeiro, fevereiro, março, abril, setembro, outubro, novembro e dezembro, aos totais precipitados anual e no período entre maio e agosto, com duração mínima de 10 anos e as mais recentes disponíveis. Com base nessas séries é que se obtiveram os valores médios, em mm.

As coordenadas geográficas latitude e longitude, em graus, foram obtidas diretamente da base de dados da ANA; a altitude, em m, de cada estação, foi extraída do modelo digital de elevação de Minas Gerais, elaborado de acordo com a metodologia de Scolforo \& Carvalho (2006); referidas variáveis se constituem nos dados de entrada dos modelos.

O processo de modelagem estatística desenvolvido consiste no ajuste de modelos lineares, por meio de regressão múltipla; para isto se elaboraram variáveis provenientes da combinação das coordenadas geográficas e altitude, as quais foram selecionadas pelo procedimento "Backward" de regressão múltipla, que avalia todas as variáveis dentro de uma regressão, excluindo automaticamente aquelas que, para determinado modelo, foram não significativas pelo teste $t$ de Student e não promoveram melhoria do coeficiente de determinação ajustado, significando que o procedimento manterá, no modelo, as variáveis não significativas, porém que colaboram com a qualidade final dos ajustes (D’Agostino, 1986; Ferreira, 2005). O programa SAS for Windows foi aplicado para ajuste dos modelos (SAS, 1985), cuja estrutura matemática é a seguinte:

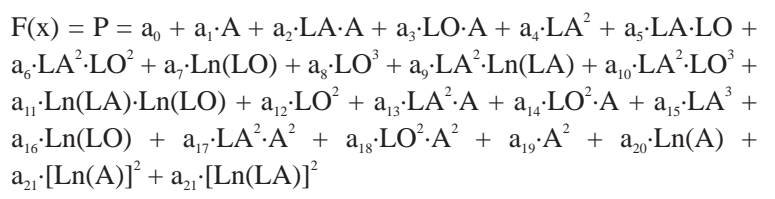

em que: $\mathrm{P}$ é a precipitação média mensal, anual ou do período seco, em mm, LA, LO e A são, respectivamente, latitude, longitude, ambas em graus, e altitude, em m; $\mathrm{a}_{0}$, $\mathrm{a}_{1}$ etc, parâmetros de ajuste do modelo estimados pela regressão. 


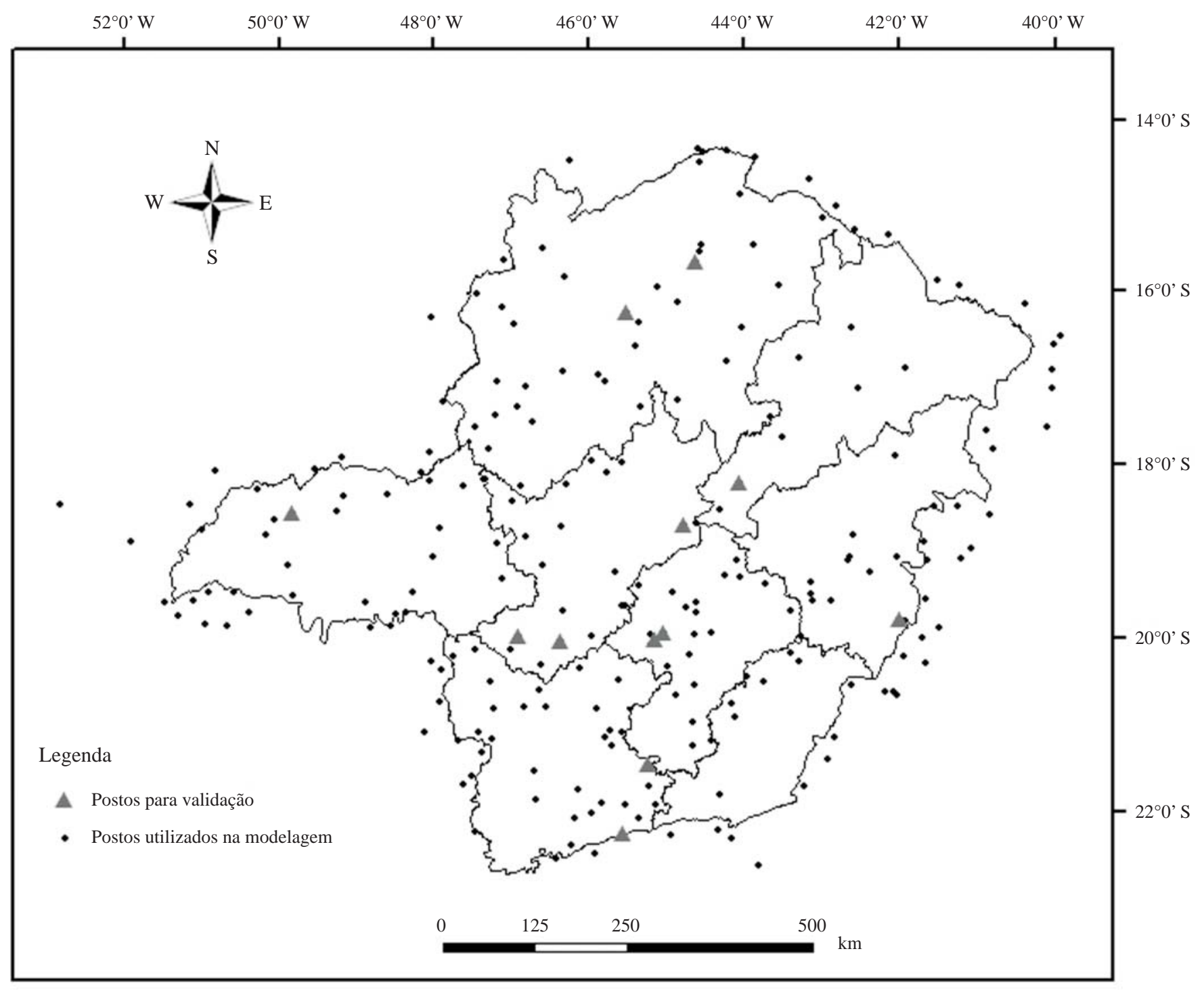

Figura 1. Estações pluviométricas de Minas Gerais e seu entorno, utilizadas na modelagem e validação dos modelos ajustados

Ressalta-se que apenas uma parte das variáveis da Eq. 1 é que comportará o modelo final, de acordo com o procedimento "Backward", mencionado antes.

Para avaliação dos modelos foram aplicados o coeficiente de determinação ajustado, o erro absoluto médio (\%), a tendência dos modelos para estimativa da precipitação em relação à média (\%) e a significância dos parâmetros estimados. O erro médio absoluto e a tendência, ambos em percentagem, foram calculados, respectivamente, por:

$$
\begin{aligned}
& \mathrm{E}(\%)=\left(\sum_{\mathrm{i}=1}^{\mathrm{n}} \frac{\left|\mathrm{V}_{\mathrm{pi}}-\mathrm{V}_{\mathrm{oi}}\right|}{V_{0 i}} \cdot 100\right) \cdot \frac{1}{\mathrm{n}} \\
& \mathrm{T}(\%)=\left(\frac{\sum_{\mathrm{i}=1}^{\mathrm{n}}\left(\mathrm{V}_{\mathrm{pi}}-\mathrm{V}_{\mathrm{oi}}\right)}{\mathrm{n}}\right) \cdot \frac{1}{\overline{\mathrm{X}}} \cdot 100
\end{aligned}
$$

em que: E é o erro médio (\%), T é a tendência dos valores estimados em relação à média (\%), $\mathrm{V}_{\mathrm{pi}}$ e $\mathrm{V}_{\mathrm{oi}}$, são, respectivamente, os valores preditos e observados e n corresponde ao número de dados trabalhados, ou seja, de estações pluvi- ométricas; além desses parâmetros estatísticos, gráficos de dispersão dos valores estimados em torno da reta 1:1 foram construídos para análise da precisão das estimativas, além de auxílio na análise de tendência dos valores estimados.

Os parâmetros estatísticos de validação foram avaliados tendo-se como referência, em primeiro instante, a base de dados aplicada no ajuste dos modelos (197 estações) e, para sua validação final, 12 estações foram excluídas da modelagem para se lhes aplicar os modelos, consistindo de uma etapa fundamental para caracterização da precisão dos mesmos. Na Figura 1 é possível distinguir, no espaço, a distribuição adotada, buscando-se distribuir as estações pluviométricas de validação pelo Estado, de forma a representar cada macrorregião do mesmo.

\section{RESULTADOS E DISCUSSÃO}

Apresentam-se, na Tabela 1, as estatísticas básicas dos dados analisados e seu estudo de correlação com as coordenadas geográficas e altitude. É oportuno destacar que o mês 
de setembro e o período seco apresentam as maiores variabilidades, de acordo com os respectivos coeficientes de variação. Para setembro, este comportamento ocorre em vista de se tratar de um mês caracterizado pela transição do período seco para o período chuvoso, razão pela qual se constatam, na série histórica, situações não esperadas e nas quais o período chuvoso se iniciou com atraso, gerando precipitação mensal praticamente nula, haja vista que a média de precipitação deste mês é de $46 \mathrm{~mm}$, típico das características climáticas do Sudeste do Brasil, sobre o qual há influência importante de fenômenos globais de circulação, especialmente do El Niño e La Niña, que promovem, respectivamente, menor volume de chuvas e menores temperaturas, com alteração relevante do regime hidrológico regional (Moreira, 1999); situação semelhante também ocorre com a precipitação no período seco que abrange, efetivamente, os meses de maio a agosto, havendo variabilidade tanto temporal quanto espacial, sobretudo esta última, provocada por diferentes características climáticas dentro do Estado de Minas Gerais. Segundo Mello et al. (2007) próximo às grandes cadeias de montanhas do Estado há ocorrência de precipitação de forma mais significativa, em particular na região Sul; outro detalhe que merece destaque diz respeito à magnitude das precipitações nesse período. Como os valores são baixos (86 mm em média), uma pequena alteração nos mesmos pode representar variabilidade significativa, impactando nos valores do coeficiente de variação.

Tabela 1. Parâmetros estatísticos básicos da precipitação no Estado de Minas Gerais e correlação com as coordenadas geográficas e altitude

\begin{tabular}{|c|c|c|c|c|c|}
\hline \multirow{2}{*}{ Período } & \multirow{2}{*}{$\begin{array}{l}\text { Média } \\
(\mathrm{mm})\end{array}$} & \multirow{2}{*}{$\begin{array}{l}\text { C.V. } \\
(\%)\end{array}$} & \multicolumn{3}{|c|}{ Coeficiente de Correlação } \\
\hline & & & Lat. & Long. & Altitude \\
\hline Anual & 1299,48 & 19,08 & $0,656^{* *}$ & $0,428^{*}$ & $0,627^{* *}$ \\
\hline Janeiro & 239,33 & 23,77 & $0,601^{*}$ & $0,477^{*}$ & $0,641^{* *}$ \\
\hline Fevereiro & 163,50 & 31,52 & $0,595^{\star}$ & $0,65^{\star *}$ & $0,637^{* *}$ \\
\hline Março & 154,56 & 24,30 & $0,523^{*}$ & $0,533^{*}$ & $0,569 *$ \\
\hline Abril & 68,25 & 25,47 & $0,329^{\text {ns }}$ & $0,359^{\text {ns }}$ & $0,262^{\text {ns }}$ \\
\hline Setembro & 46,06 & 39,29 & 0,694 ** & $0,238^{\text {ns }}$ & $0,310^{\mathrm{ns}}$ \\
\hline Outubro & 106,83 & 22,46 & $0,605^{\star}$ & $0,305^{\text {ns }}$ & 0,520 * \\
\hline Novembro & 186,76 & 17,23 & $0,145^{\mathrm{ns}}$ & $-0,042^{\mathrm{ns}}$ & $0,360^{\mathrm{ns}}$ \\
\hline Dezembro & 250,43 & 20,65 & $0,379^{\text {ns }}$ & $0,260^{\mathrm{ns}}$ & $0,570^{*}$ \\
\hline P. Seco & 85,96 & 56,07 & $0,426^{*}$ & $-0,109^{n s}$ & $0,0139^{\text {ns }}$ \\
\hline
\end{tabular}

** Significativo a $1 \%$ de probabilidade; * Significativo a $5 \%$ de probabilidade; ns - não significativo; CV - coeficiente de variação; Lat - Latitude; Long - Longitude

No contexto da correlação estatística dos totais precipitados em função das coordenadas geográficas e altitude, verifica-se que o total precipitado médio do mês de abril apresentou coeficientes de correlação baixos e não significativos, tal como o mês de novembro; para o período seco, apenas a latitude se mostrou significativa. Conforme Ferreira (2005), coeficientes de correlação baixos e pouco significativos poderão influenciar no ajuste dos modelos reduzindo suas qualidades estatísticas. No contexto da precipitação, a latitude e a altitude proporcionaram coeficientes elevados e, em alguns casos, altamente significativos, mostrando que é possível modelar adequadamente os totais precipitados em função das coordenadas geográficas e altitude; desta forma, quanto maiores a latitude e a altitude, maiores também serão os totais precipitados, reflexo das características climáticas e influência orográfica no comportamento pluvial das regiões do Estado onde, sob maiores latitudes, há predominância de climas mais frios, do tipo Cwb/Cwa pela classificação de Köppen (Antunes, 1986); essas regiões recebem a influência de frentes frias com maior intensidade, as quais enfraquecem sempre que adentram no Estado em direção às menores latitudes, significando que eventos de chuva do tipo frontal, mais comuns durante o inverno, são mais significativos e importantes para regiões de maiores latitudes, mostrando sua importância na precipitação total do período seco (Vianello \& Alves, 2000).

Os modelos ajustados para as precipitações médias mensais de janeiro, fevereiro, março e abril e precipitação média anual, estão apresentados na Tabela 2, em que constam os parâmetros estimados para cada variável selecionada pelo procedimento "Backward”, com as respectivas significâncias estatísticas pelo teste de t de "Student", coeficiente de determinação ajustado, erro médio (\%) e tendência das estimativas em relação à média (\%). Em diversos modelos se verificam parâmetros estimados não significativos; contudo, eles foram mantidos no modelo final visto que sua retirada acarretou redução do coeficiente de determinação procedimento este que, segundo Ferreira (2005) e D’Agostino (1986), deve ser adotado sem prejuízo significativo para a qualidade final dos modelos, uma vez que os graus de liberdade do erro são consideravelmente altos.

Analisando-se os modelos para precipitação anual e para os meses de janeiro e fevereiro, verificam-se modelos com boas qualidades estatísticas, com coeficientes de determinação ajustados significativos e elevados $(>0,75)$. Os erros médios também se apresentam baixos para modelagens desta natureza, além de não mostrarem, praticamente, nenhuma tendência em relação à média $(<2 \%)$. Marquínez et al. (2003), modelando as precipitações anuais e nos períodos seco e úmido para a região da Cantabria (Espanha), ajustaram modelos estatísticos pelo procedimento "Stepwise", com coeficientes de determinação menores que 0,67. Os autores ajustaram modelos com 15 parâmetros e um número maior e mais complexo de variáveis de entrada necessárias para sua aplicação (5 variáveis topográficas e geográficas contra 3 deste estudo). Comparando-se o modelo para precipitação anual ajustado pelos autores ao ajustado para Minas Gerais, verifica-se apreciável ganho de qualidade estatística e de precisão por este último, com coeficiente de determinação ajustado de 0,756 contra 0,670 e erro médio de 8 contra $10 \%$, além de variáveis de entrada de fácil obtenção.

Os modelos estimados para os meses de março e abril apresentam redução considerável de qualidade, especialmente para o mês de abril, com coeficiente de determinação de 0,513 e erro médio praticamente o dobro daquele ajustado para o período anual; no entanto, os erros médios e as tendências são baixos o suficiente para caracterização dos modelos como aceitáveis e com boa precisão (Daly et al., 1994; Goodale et al., 1998; Marquínez et al., 2003). O comportamento desses modelos pode ser explicado de forma semelhante ao mês de setembro, uma vez que o mês de abril representa uma transição 
do período chuvoso para o seco, além de apreciável redução das temperaturas do ar, em especial nas regiões sob clima Cwb/ Cwa, situação que, normalmente, acarreta em aumento da variabilidade dos totais precipitados, dificultando a obtenção de uma relação causa-efeito mais significativa. Os coeficientes de correlação para abril, mostrados na Tabela 1, atestaram este comportamento.

Daly et al. (1994), modelando variáveis climáticas em função das coordenadas geográficas, também obtiveram modelos com características estatísticas de precisão semelhantes, com $\mathrm{R}^{2}$ ajustado máximo de 0,60 e erros da ordem de $10 \%$, concluindo que os modelos podem ser aplicados, uma vez que erros desta magnitude são baixos, em se tratando da modelagem de variáveis físicas ambientais cuja variabilidade é naturalmente alta devido ao grande número de atributos envolvidos na sua dinâmica, cuja inclusão em um modelo estatístico tornaria sua aplicação prática bastante complexa.

Tabela 2. Modelos desenvolvidos para as precipitações correspondentes aos meses de janeiro a abril e anual e parâmetros para análise de suas qualidades estatísticas

\begin{tabular}{|c|c|c|c|c|c|}
\hline Variável & Anual & Janeiro & Fevereiro & Março & Abril \\
\hline Intercepto & $101077^{\mathrm{ns}}$ & $-400244^{\star}$ & $-314694^{\star *}$ & $-627,4^{\text {ns }}$ & $35939 * *$ \\
\hline A & $-1,30^{\text {ns }}$ & $-6,44^{\star}$ & - & - & - \\
\hline $\mathrm{LA} * \mathrm{~A}$ & $0,028^{\text {ns }}$ & - & - & $-0,034^{\star *}$ & $0,0201^{* *}$ \\
\hline$L 0^{\star} \mathrm{A}$ & $0,0244^{\text {ns }}$ & $0,296^{*}$ & - & - & $-0,0136^{\star *}$ \\
\hline $\mathrm{LA}^{2}$ & $349,15^{*}$ & - & - & - & 1,266 ** \\
\hline $\mathrm{LA} * \mathrm{~L} 0$ & $-85,50^{\star}$ & $125,04^{*}$ & $159,16^{\star *}$ & $13,80^{*}$ & $-1,605^{\star \star}$ \\
\hline $\mathrm{LA}^{2 *} \mathrm{LO}^{2}$ & $0,151^{* *}$ & - & $-0,0273^{* *}$ & $-0,0037^{\star}$ & - \\
\hline $\operatorname{Ln}(\mathrm{LO})$ & -28296 ** & - & - & - & -11954 ** \\
\hline $\mathrm{LO}^{3}$ & $0,263^{\star *}$ & $0,309 * *$ & - & - & $-0,0743^{* *}$ \\
\hline $\mathrm{LA}^{2 *} \operatorname{Ln}(\mathrm{LA})$ & $-109,3^{*}$ & $-38,77^{*}$ & $-46,33^{\star *}$ & $-0,368 * *$ & - \\
\hline $\mathrm{LA}^{2 *} \mathrm{LO}^{3}$ & $-0,002^{\star *}$ & $-2,85 \mathrm{E}-4^{\star *}$ & - & - & - \\
\hline $\operatorname{Ln}(\operatorname{LA})^{\star} \operatorname{Ln}(\mathrm{L} 0)$ & - & $-52330 *$ & $-57435^{\star \star}$ & $-3598,54^{\star *}$ & - \\
\hline $\mathrm{LO}^{2}$ & - & $-33,90^{\star *}$ & $-6,79 * *$ & - & $8,264^{* *}$ \\
\hline $\mathrm{LA}^{2 *} \mathrm{~A}$ & - & - & $1,44 \mathrm{E}-4^{* *}$ & $0,002^{\star *}$ & - \\
\hline $\mathrm{LO}^{2 *} \mathrm{~A}$ & - & $-0,0034^{*}$ & - & - & $1,088 \mathrm{E}-4^{*}$ \\
\hline $\mathrm{LA}^{3}$ & - & $2,405^{\star}$ & $3,342^{\star *}$ & - & - \\
\hline$A^{3}$ & - & $-1,45 \mathrm{E}-8^{\mathrm{ns}}$ & - & - & - \\
\hline $\operatorname{Ln}(\mathrm{LA})$ & - & $158259 *$ & $169022^{* *}$ & $8143,63^{\star *}$ & - \\
\hline $\operatorname{Ln}(\mathrm{LO})$ & - & $128360^{*}$ & 101096 ** & - & - \\
\hline $\mathrm{LA}^{2 *} \mathrm{~A}^{2}$ & - & - & - & $-5,3 \mathrm{E}-7^{\star *}$ & $-3,90 \mathrm{E}-7^{* *}$ \\
\hline $\mathrm{LO}^{2 *} \mathrm{~A}^{2}$ & - & - & - & $9,65 \mathrm{E}-8^{* *}$ & $8,33 \mathrm{E}-8^{* *}$ \\
\hline$[\operatorname{Ln}(\operatorname{LO})]^{2}$ & - & - & - & $581,65^{\star}$ & - \\
\hline$[\operatorname{Ln}(\operatorname{LA})]^{2 *}[\operatorname{Ln}(\mathrm{LO})]^{2}$ & - & - & - & - & $4,494^{*}$ \\
\hline $\mathrm{R}_{\text {ajustado }}^{2}$ & 0,756 & 0,776 & 0,802 & 0,658 & 0,513 \\
\hline Erro médio (\%) & 7,97 & 9,14 & 10,79 & 10,85 & 14,31 \\
\hline Tendência (\%) & 1,42 & $-1,88$ & 1,44 & $-1,27$ & 1,04 \\
\hline
\end{tabular}

Os modelos ajustados para os meses de setembro a dezembro e período seco, estão apresentados na Tabela 3; na Tabela 2, eles são semelhantes aos ajustados para os meses de março e abril, porém apresentam maiores tendências nas estimativas, sobretudo nos meses de outubro, novembro e período seco, verificando-se comportamento de superestimativa dos valores; na Figura 2 pode-se verificar a dispersão dos valores em torno da reta 1:1 e, para esses modelos (le- tras g, h, j), se nota nitidamente acúmulo de pontos acima da reta $1: 1$, caracterizando tendência de superestimativa dos valores de precipitação média, principalmente no modelo para o período seco que, em média, tende a superestimar o total precipitado em aproximadamente $31 \mathrm{~mm}$; tal comportamento mostra que o mesmo deve ser aplicado com ressalva, em virtude da sua menor confiabilidade estatística. Os demais gráficos de dispersão mostram as boas qualidades estatísticas dos demais modelos, com baixa dispersão dos valores e sem acúmulo significativo de pontos, acima ou abaixo da reta $1: 1$.

Tabela 3. Modelos para precipitações correspondentes aos meses de setembro a dezembro e período seco e parâmetros para análise de suas qualidades estatísticas

\begin{tabular}{|c|c|c|c|c|c|}
\hline Variável & Setembro & Outubro & Novembro & Dezembro & $\begin{array}{l}\text { Período } \\
\text { Seco }\end{array}$ \\
\hline Intercepto & $889,83^{\text {ns }}$ & $44538^{* *}$ & $-54015^{\star *}$ & $-93642^{\star \star}$ & 114030 ** \\
\hline A & $-2,29 *$ & $4,248^{\star}$ & - & $-6,077^{\star}$ & $-8,52^{\star \star}$ \\
\hline $\mathrm{LA} A \mathrm{~A}$ & $0,071^{\text {** }}$ & $-0,128$ ** & - & - & $0,2352^{* *}$ \\
\hline $\mathrm{LO} \mathrm{A}_{\mathrm{A}}$ & $0,0697^{*}$ & $-0,181$ * & $-3 \mathrm{E}-4^{\mathrm{ns}}$ & $0,279^{*}$ & $0,2708^{* *}$ \\
\hline $\mathrm{LA}^{2}$ & - & $6,94^{\star \star}$ & - & $113,83^{\star *}$ & - \\
\hline $\mathrm{LA} * \mathrm{~L} 0$ & $-11,90^{\star \star *}$ & - & $-3,153^{*}$ & $-25,80$ ** & $-39,351^{\star *}$ \\
\hline $\mathrm{LA}^{2 *} \mathrm{LO}^{2}$ & $0,0158^{* *}$ & - & 0,0149 * & $0,0357^{* *}$ & $0,032^{\star \star}$ \\
\hline $\operatorname{Ln}(\mathrm{L} 0)$ & - & - & - & 32120 * & - \\
\hline $\mathrm{LO}^{3}$ & $0,045^{\star \star}$ & $-0,1139 * \star$ & $0,187^{\text {** }}$ & $0,3018^{\star *}$ & $-0,171^{\star \star}$ \\
\hline $\mathrm{LA}^{2 \star} \operatorname{Ln}(\mathrm{LA})$ & - & $-1,925^{\star *}$ & - & $-33,66$ ** & - \\
\hline $\mathrm{LA}^{2 *} \mathrm{LO}^{3}$ & $-1,804 \mathrm{E}-4$ * & $-5,04 \mathrm{E}-6$ ** & $-2,19 \mathrm{E}-4^{*}$ & $-4,32 \mathrm{E}-4 * *$ & $-3,16 \mathrm{E}-4^{*}$ \\
\hline $\operatorname{Ln}(\mathrm{LA})^{\star} \operatorname{Ln}(\mathrm{L} 0)$ & - & - & - & - & $3221,03^{* *}$ \\
\hline $\mathrm{LO}^{2}$ & $-1,821^{*}$ & $11,52^{\star \star}$ & $-16,71 * *$ & $-25,205^{\star \star}$ & 24,156 ** \\
\hline $\mathrm{LA}^{2 \star A} \mathrm{~A}$ & $-0,00183^{*}$ & $0,00441^{* *}$ & - & - & $-0,0059 * *$ \\
\hline $\mathrm{LO}^{2 *} \mathrm{~A}$ & $-7,47 \mathrm{E}-4^{*}$ & 0,00209* & - & $-0,0032^{*}$ & $-0,0029 *$ \\
\hline $\mathrm{LA}^{3}$ & $-0,193^{\star \star}$ & - & $-0,231$ ** & - & $-0,216^{\star}$ \\
\hline $\operatorname{Ln}(\mathrm{L} 0)$ & - & $-15010^{* \star}$ & 18796 ** & 32120 ** & -42360 ** \\
\hline $\mathrm{LA}^{2 \star} \mathrm{A}^{2}$ & - & $-7,97 \mathrm{E}-7$ ** & - & $-1,651 \mathrm{E}-7^{\mathrm{ns}}$ & - \\
\hline $\mathrm{LO}^{2 *} \mathrm{~A}^{2}$ & - & - & - & $3,90 \mathrm{E}-8^{\mathrm{ns}}$ & - \\
\hline$A^{2}$ & - & $4,70 \mathrm{E}-4$ ** & $3,063 \mathrm{E}-5^{*}$ & - & - \\
\hline $\operatorname{Ln}(A)$ & - & $-268,49 *$ & - & - & - \\
\hline$[\operatorname{Ln}(A)]^{2}$ & $535,58^{\star}$ & $34,54^{\star}$ & - & - & - \\
\hline $\mathrm{R}^{2}$ & 0,756 & 0,651 & 0,517 & 0,653 & 0,510 \\
\hline Erro médio (\%) & 15,62 & 16,64 & 14,71 & 10,25 & 30,19 \\
\hline Tendência (\%) & $-2,04$ & 16,57 & 14,85 & $-3,03$ & 36,20 \\
\hline
\end{tabular}

Apresentam-se, na Figura 3, os erros médios e as tendências produzidas pelo processo de validação dos modelos. Esta situação é extremamente importante para validação final dos modelos pois consiste da sua aplicação a uma base de dados desconhecida do processo de modelagem. Em termos de erro médio, valores consideravelmente baixos (<20\%) são observados para os modelos mensal e anual; para o período seco, o erro foi superior a 30\%, acompanhado de tendência de superestimativa superior a $40 \%$ em relação à média, reforçando as observações desenvolvidas sobre este modelo. Dentre os modelos ajustados por Marquínez et al. (2003), aquele associado ao período seco também apresentou as menores estatísticas de precisão sendo, inclusive, o modelo com o maior número de parâmetros estimados, produzindo menor grau de liberdade e demonstrando que a modelagem para esta 
A.

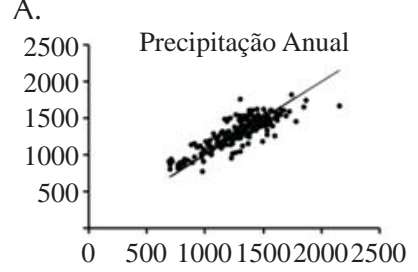

C.

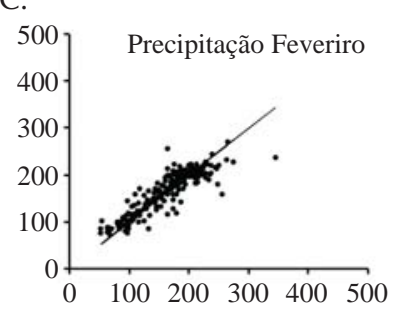

E.
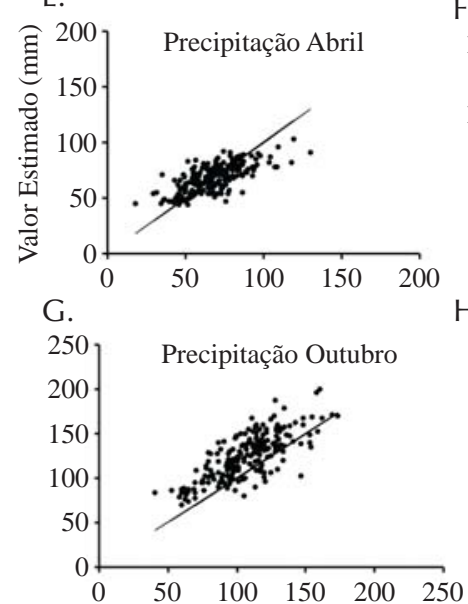

I. J.

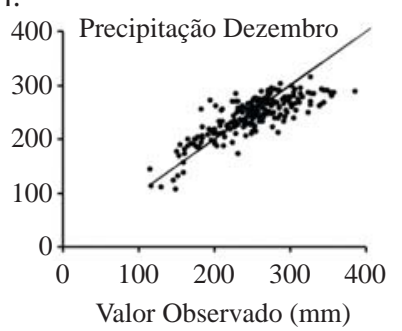

B.

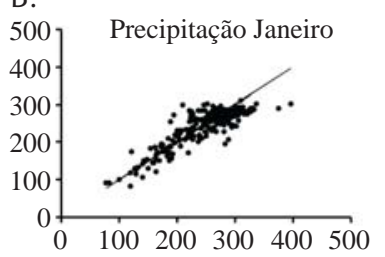

D.

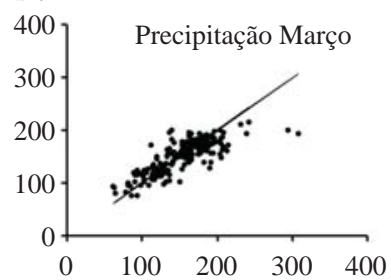

F.

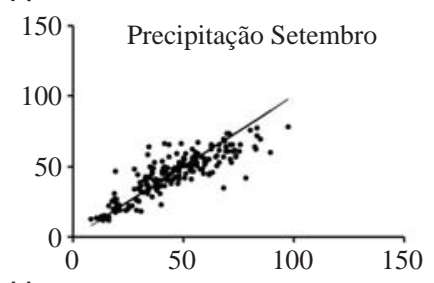

$\mathrm{H}$.
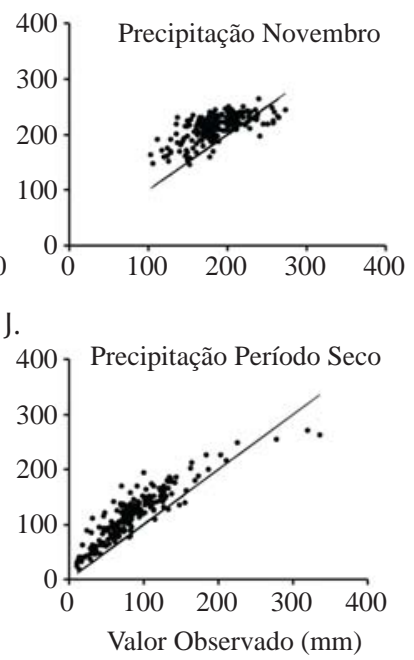

$\overline{\text { Figura 2. Dispersão dos valores preditos pelos modelos ajustados em torno }}$ da reta $1: 1$

situação tende a apresentar maior dificuldade, porém a tendência gerada pelo modelo de Marquínez et al. (2003) é inferior à gerada pelo correspondente modelo obtido por este trabalho mostrando que, para o período seco, é conveniente a aplicação de outras variáveis, especialmente associadas ao relevo, para geração de um modelo de melhor qualidade. Essas variáveis de entrada, de acordo com Marquínez et al. (2003), podem ser: a menor distância de cada localidade de interesse ao litoral, a menor distância em relação a elementos topográficos importantes, como serras e montanhas de grande expressão (Serra da Mantiqueira, Serra do Espinhaço, Serra da Canastra e outras relevantes dentro do Estado de Minas Gerais), declividade média da região, dentre outros; no entanto, para aplicação do modelo haverá dificuldades significativas para obtenção das variáveis de entrada, limitando seu uso.

Ainda com referência à Figura 3, nos modelos para o período anual, fevereiro, abril e dezembro, a tendência é praticamente nula; para janeiro, março e setembro, ela é de subestimativa, apesar de pouco expressiva (menor que $10 \mathrm{~mm}$ para março e $5 \mathrm{~mm}$ para setembro), enquanto para outubro e novembro, a tendência é de superestimativa, isto é, da ordem de $10 \%$ (menor que $11 \mathrm{~mm}$ para outubro e $19 \mathrm{~mm}$ para novembro). Marquínez et al. (2003) encontraram, no processo de validação dos modelos, erros da ordem de 14\% para precipitação anual e tendências semelhantes, concluindo sobre a validade dos mesmos para aplicação prática; desta maneira, é possível constatar o melhor desempenho dos modelos ajustados por este trabalho permitindo que sejam aplicados para estimativa dos totais precipitados, representando importante ferramenta para o manejo técnico-ambiental do Estado de Minas Gerais.

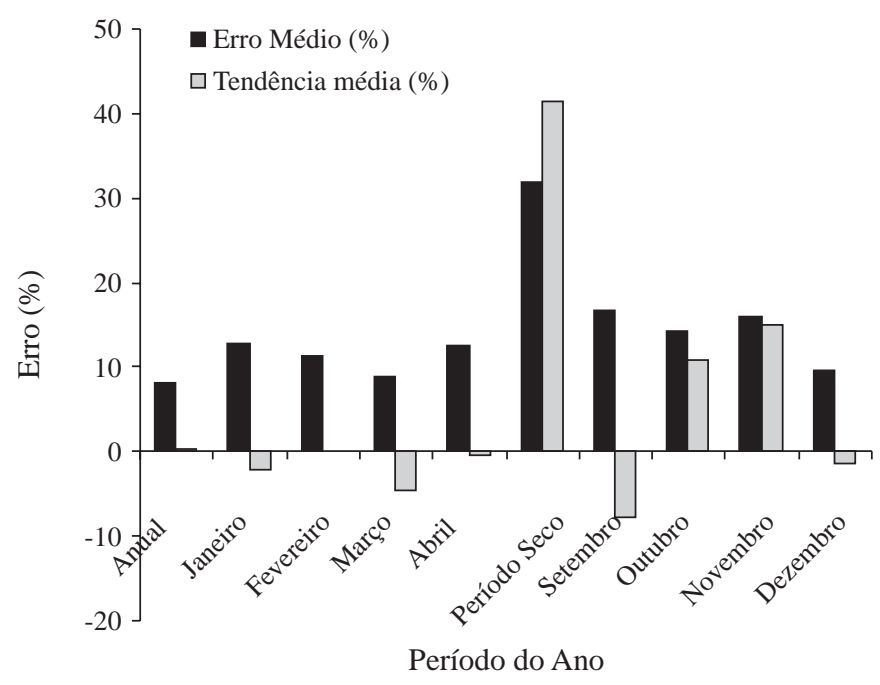

Figura 3. Erros e tendências médias de estimativa dos modelos ajustados com base no processo de validação

\section{CONCLUSÕES}

1. Os modelos ajustados para estimativa das precipitações totais médias mensais e anual apresentaram parâmetros estatísticos que os caracterizam como de boa qualidade, podendo ser aplicados para estimativa da precipitação média no Estado de Minas Gerais.

2. O modelo para estimativa da precipitação no período seco mostrou-se eficiente; contudo, produziu tendência de superestimativa, situação esta que não o desqualifica devido à dificuldade de ajuste de modelos desta natureza para o período seco, considerando-se apenas as coordenadas geográficas e altitude como dados de entrada.

3. Para obtenção de um modelo para o período seco com o mesmo padrão de qualidade dos demais, outras variáveis de entrada, associadas ao relevo, devem ser incorporadas à modelagem, em virtude das características orográficas de Minas Gerais, tornando mais complexa a obtenção dos dados de entrada e aplicação do modelo. 


\section{LITERATURA CITADA}

Antunes, F. Z. Caracterização climática do estado de Minas Gerais: Climatologia agrícola. Informe Agropecuário, v.12, p.9-13, 1986.

D’Agostino, R. B. Tests for the normal distribution. In: D’Agostino, R. B.; Stephen, M. A. (ed.). Goodness-of-fit techniques. New York: Marcel Dekker, 1986, p.367-419.

Daly, C.; Neilson, R. P.; Philips, D. L. A statistical-topographic model for mapping climatological precipitation over mountainous terrain. Journal of Applied Meteorology, v.33, n.2, p.140-158, 1994.

Ferreira, D. F. Estatística básica. Lavras: UFLA, 2005. 760p.

Goodale, C. L.; Alber, J. D.; Ollinger, S. V. Mapping monthly precipitation, temperature and solar radiation for Ireland with polynomial regression and digital elevation model. Climate Research, v.10, n.1, p.35-49, 1998.

Marquínez, J.; Lastra, J.; Garcia, P. Estimation models for precipitation in mountainous regions: the use of GIS and multivariate analysis. Journal of Hydrology, v.270, n.1, p.1-11, 2003.

Martinez-Cob, A. Multivariate geostatistical analysis of evapotranspiration and precipitation in mountainous terrain. Journal of Hydrology, v.174, n.1-2, p.19-35, 1996.

Mello, C. R.; Sá, M. A. C.; Curi, N.; Mello, J. M.; Viola, M. R.; Silva, A. M. Erosividade mensal e anual da chuva no Estado de Minas Gerais. Pesquisa Agropecuária Brasileira, v.42, n.4, p.537-545, 2007.
Moreira, A. A. M. A influência da circulação de macroescala sobre o clima de Belo Horizonte: Estudo sobre as possíveis influências do fenômeno El Niño sobre o clima local. Belo Horizonte: UFMG, 1999. 186p. Dissertação Mestrado

Scolforo, J. R. S.; Carvalho, L. M. T. Mapeamento e inventário da flora nativa e dos Reflorestamentos de Minas Gerais. Lavras: IEF/UFLA, 2006. 288p.

SAS - Statistical Analyses System. Language guide for personal computers. 6.ed. Cary: SAS Institute, 1985. 429p.

Sediyama, G.; Mello Jr., J. C. Modelos para estimativas das temperaturas normais mensais médias, máximas, mínimas e anual no estado de Minas Gerais. Revista Engenharia na Agricultura, v.6, n.1, p.57-61, 1998.

Vianello, R. L.; Alves, A. R. Meteorologia básica e aplicações. Viçosa: UFV, 2000. 448p.

Viola, M. R. Comportamento espacial e temporal da chuvas intensas no Estado de Minas Gerais. Lavras: UFLA, 2006. 74p. Monografia Conclusão Curso

Weisse, A. K.; Bois, P. Topographic effects on statistical characteristics of heavy rainfall and mapping in the French Alps. Journal of Applied Meteorology, v.40, n.4, 720-740, 2001.

Wu, S. J.; Tung, Y. K.; Yang, J. C. Stochastic generation of hourly rainstorm events. Stochastic Environmental Research Risk Assessment, v.21, p.195-212, 2006. 